\title{
Lagrangian acceleration timescales in anisotropic turbulence
}

\author{
Peter D. Huck, ${ }^{1,2}$ Nathanael Machicoane, ${ }^{2}$ and Romain Volk ${ }^{1, *}$ \\ ${ }^{1}$ Univ Lyon, Ens de Lyon, Univ Claude Bernard, CNRS, Laboratoire de Physique, F-69342 Lyon, France \\ ${ }^{2}$ University of Washington, Department of Mechanical Engineering, Seattle, Washington 98195, USA
}

(Received 8 December 2018; published 10 June 2019)

\begin{abstract}
We present experimental Lagrangian measurements of tracer particle acceleration autocorrelation functions in an anisotropic and inhomogeneous flow spanning the typical range of experimentally accessible Reynolds numbers. The large-scale forcing of the flow creates a stagnation point topology where straining motion governs the anisotropic velocity and acceleration fluctuations. We show that the timescales of the acceleration components remain anisotropic at high Reynolds numbers and that they are related to the dissipative timescale by the Lagrangian structure function scaling constants $C_{0}$ and $a_{0}$, as well as $C_{0}^{\star}$, which is shown to be kinematically related to the velocity increments. The proposed scaling relation is supported by observations using experimental Lagrangian trajectory data sets and analytical calculations using a jointly Gaussian two-time stochastic model. Examination of acceleration power spectra show that acceleration fluctuations become isotropic in the dissipative range, which suggests that the acceleration timescale is determined not only by small scales, but also by large and anisotropic scales whose contributions are substantial, even in the high Reynolds number limit.
\end{abstract}

DOI: 10.1103/PhysRevFluids.4.064606

\section{INTRODUCTION}

Anisotropy is an inescapable aspect of nearly all canonical turbulent configurations and may arise by different mechanisms coupled to the presence of rotation, shear, or strain [1]. In particular, a strong fundamental interest exists for straining flows where an initially isotropic turbulence is driven to anisotropy. The application of steady strain in a wind-tunnel flow by means of an axisymmetric converging duct in order to attenuate longitudinal velocity fluctuations [2] was originally theorized by Prandtl as the spanwise contraction of cylindrical vortices which diminish streamwise velocity fluctuations by conservation of angular momentum. From subsequent theoretical analyses [3,4] as well as experimental and direct numerical simulations (DNSs) the role of strain may be summarized by two essential points [5-11]. First, the large energy-containing scales experience an amplification of velocity fluctuations in the contracting direction and an attenuation in the elongating direction. Second, distortion at large scales leads to deviation from local isotropy in the rate of strain tensor.

Particle tracking velocimetry (PTV) [12-14] and DNSs [15-17] permit the investigation of Lagrangian statistics and the attendant influence of shear and strain. The latter was addressed, for instance, in the von Kármán configuration, where two counter-rotating disks act as centrifugal pumps to impose a stagnation point $[18,19]$ and turbulence production that is governed by the accompanying straining motion [20] in the central region. Velocity fluctuations display a strong, Reynolds-number-dependent, anisotropy with the dominant component aligned with the contracting direction [20-22]. Based on energy flux arguments in Kolmogorov similarity theory (K41), velocity increments follow a diffusive scaling $D_{2}^{L}(\tau)=\left\langle[v(t+\tau)-v(t)]^{2}\right\rangle \simeq C_{0} \varepsilon \tau$ for time lags $\tau$ in the

*romain.volk@ens-lyon.fr 
Lagrangian inertial range where $\varepsilon$ is the turbulent dissipation rate [23]. Single component values of the nondimensional constant $C_{0}$ have been reported in a review of published results [24].

Measurements of $C_{0}$ performed in anisotropic turbulence were also found to be strongly anisotropic, reflecting the large-scale straining structure of the flow [21], and were observed to slowly increase with Reynolds number. An asymptotic extrapolation of the data [21] based on results from stochastic modeling [25] suggests that anisotropy persists for arbitrarily large Reynolds numbers. In particular, the components corresponding to the axisymmetrically contracting direction were larger than in the extensional direction, in broad agreement with the tendencies of the large, energy-containing scales of strained wind tunnel turbulence. Flow anisotropy was found to be smaller in the dissipative scale range $[22,26]$, studied by investigating the acceleration variance of Lagrangian tracers $\left\langle a^{2}\right\rangle$, which follows the Heisenberg-Yaglom [27,28] scaling $\left\langle a^{2}\right\rangle \simeq a_{0} \varepsilon^{3 / 2} v^{-1 / 2}$ where $v$ is the kinematic viscosity of the fluid and $a_{0}$ an increasing function of the Reynolds number. As opposed to observations in the inertial range, anisotropy in the component values of $a_{0}$ $[22,29]$ has been reported to be a decreasing function of Reynolds numbers with some experiments displaying nearly isotropic values [30].

Two-time statistics of acceleration measured using the acceleration autocorrelation function, $R_{a}(\tau)=\langle a(t) a(t+\tau)\rangle /\left\langle a^{2}\right\rangle$, have received less attention than one-time acceleration statistics such as acceleration variance. It is known that acceleration is correlated over short timescales, with a zero crossing $t_{0} \approx 2 \tau_{\eta}=2 \sqrt{v / \varepsilon}$ such that $R_{a}\left(t_{0}\right)=0$, as observed in both numerical simulations [31] and experiments [22,32-34], but little information has been reported on its anisotropy. In the strain-dominated region of the von Kármán flow, it was observed that the zero-crossing time $t_{0} \approx 3 \tau_{\eta}$ was larger in the strongly fluctuating, contracting direction than $t_{0} \approx 2.2 \tau_{\eta}$ measured along the elongating direction, similar to the observations in DNSs of homogeneous and isotropic turbulence (HIT) $[31,35,36]$. It is of note that the tendency of the strongest fluctuating acceleration component to have the most prolonged correlation has also been reported experimentally in a turbulent channel flow [37] and in rotating turbulence [38].

To the authors' knowledge, no explanation has been given for the observed anisotropy of acceleration timescales, and no information on its evolution with the Reynolds number is available. In this article we address dissipative timescale anisotropy in a turbulent flow where a mean straining motion strongly influences the turbulence. We show that the timescales of acceleration components, measured as $\tau_{a}=\int_{0}^{t_{0}} R_{a}(\tau) d \tau$, remain anisotropic at high Reynolds numbers and are well predicted by the relation $\tau_{a}=C_{0}^{\star} \tau_{\eta} / 2 a_{0}$ where $C_{0}^{\star}$ is a new scaling constant analogous to $C_{0}$. This scaling relation is supported both by observations using data sets of Lagrangian trajectories obtained in two different von Kármán flows spanning a wide range of Reynolds numbers and by analytical calculation using the jointly Gaussian two-time stochastic model of Sawford [25]. Although this relation implies that acceleration timescales will remain anisotropic in the high Reynolds number limit, examination of acceleration power spectra show that acceleration fluctuations become isotropic in the dissipative range. This indicates that statistical quantities such as the acceleration variance and correlation timescale not only depend on small scales but are influenced by larger scales which are anisotropic.

\section{EXPERIMENTAL SETUP}

The Lagrangian turbulence data presented here combine the results of two measurement techniques: extended laser Doppler velocimetry (eLDV) [33,34] and shadow particle tracking velocimetry (SPTV) [20,39,40]. In both cases the experiments were performed in a von Kármán flow [Figs. 1(a) and 1(b)] where an intense turbulence is created between two counter-rotating disks of diameter $D=14.2 \mathrm{~cm}$, with straight blades separated by a distance $H=20 \mathrm{~cm}$. The flow is bounded by a cylindrical vessel whose length is $L=25 \mathrm{~cm}$ and width is $W=15 \mathrm{~cm}$. In the case of the eLDV experiments the cylinder has a round cross section [Fig. 1(a)], while the SPTV experiments were carried out in a square cross section [Fig. 1(b)]. Both configurations share properties in that the differential rotation of the disks establishes the large-scale flow and also acts as a centrifugal pump 

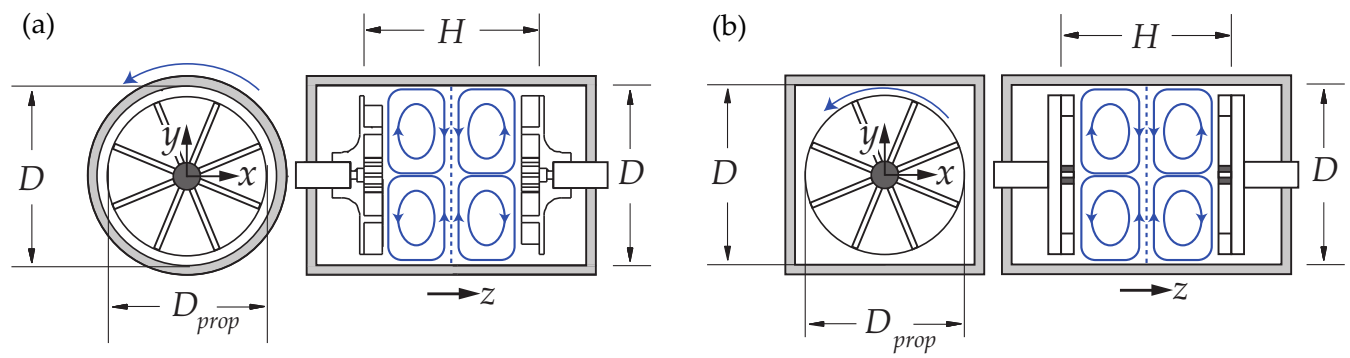

FIG. 1. Experimental setup. (a) Round geometry of the von Kármán flow measured with extended laser Doppler velocimetry (eLDV). (b) Square geometry of the von Kármán flow measured with shadow particle tracking velocimetry (SPTV).

whose recirculation pattern creates a stagnation point topology in the central region (Fig. 1) where the straining motion is responsible for the high degree of anisotropy observed [20]. Due to the geometry of the vessel and its forcing, each configuration is nearly axisymmetric in the stagnation point region, and the use of eLDV and SPTV gives complementary measurements of the axial $(z)$ and radial $(x)$ components of two similar flows over a broad range of Reynolds numbers.

Due to the geometry of the vessel and its forcing, each configuration is nearly axisymmetric in the stagnation point region, and the use of eLDV and SPTV gives complementary measurements of the axial $(z)$ and radial $(x)$ components of two similar flows over a broad range of Reynolds numbers. All experiments were performed in the fully turbulent regime. The Taylor-scale-based Reynolds number $\operatorname{Re}_{\lambda}=\left[15 v_{\text {rms }}^{4} /(\nu \varepsilon)\right]^{1 / 2}$ was varied over the typically accessible range of laboratory Lagrangian experiments [41], where $v_{\text {rms }}$ is the root mean square (rms) velocity, $v$ is the kinematic viscosity of the fluid, and $\varepsilon$ the turbulent dissipation rate (Table I) .

In the round cross-sectional geometry, which corresponds to the highest values of the Reynolds number (Table I), the fluid is pure water, and the trajectories were obtained by means of eLDV. Lagrangian tracer trajectories for particles with diameters of $30 \mu \mathrm{m}$ (two times the dissipation scale $\eta=\left(v^{3} / \varepsilon\right)^{1 / 4}$. Such neutrally buoyant particles can be considered tracers as shown in previous work [33,42]. Temporal sampling increments $\left(d t=1 / 167 \mathrm{~ms}\right.$ for $\left.\operatorname{Re}_{\lambda}=810\right)$ were sufficient to ensure that temporal resolution and the ratio of the dissipation timescale $\left(\tau_{\eta}=(v / \varepsilon)^{1 / 2}\right)$ to temporal increment were high: $\tau_{\eta} / d t=33$. The measurements were performed in the vicinity of the geometrical center over a volume of $5 \times 5 \times 10 \mathrm{~mm}^{3}$ where the mean flow is small and the Lagrangian tracks are long enough to compute acceleration statistics. In this setup, the dissipation rate $\varepsilon$ is estimated based on the power consumption of the motors [33], which was found to be in agreement with hot-wire anemometry measurements in flows with similar geometries [43].

In the square cross-sectional geometry, corresponding to the lowest values of the Reynolds number (Table I), the fluid is a water-Ucon mixture with a viscosity of $v=8.2 \times 10^{-6} \mathrm{~m}^{2} \mathrm{~s}^{-1}$, and tracers with diameters of $251 \mu \mathrm{m}$ (2.3 times the dissipation scale) are tracked using SPTV with a temporal resolution of $d t=1 / 13 \mathrm{~ms}$ and $\tau_{\eta} / d t=18$ for $\operatorname{Re}_{\lambda}=200$. Although the temporal increment in SPTV is an order of magnitude larger than in eLDV, it is sufficient to measure the acceleration dynamics using the denoising technique from Machicoane et al. [44] (see discussion below). SPTV uses a small LED located at the focal point of a large parabolic mirror $(15 \mathrm{~cm}$ in diameter) creating a collimated light beam that illuminates particles which appear as shadows when projected onto a high-speed camera $[20,39,40]$. A second, orthogonal beam creates a redundant axis which permits reconstruction of trajectories in a large volume $6 \times 6 \times 5 \mathrm{~cm}^{3}$. As the SPTV measurement volume is much larger than in the eLDV case, we use a subset of particle trajectories which pass through a $1 \mathrm{~cm}$ diameter sphere near the stagnation point. As opposed to the round cross-sectional case, SPTV provided the velocity and acceleration at known positions so that the mean flow could be reconstructed. This permits the estimation of the turbulent dissipation rate as $\langle\vec{v} \cdot \vec{a}\rangle-\langle\vec{v}\rangle \cdot\langle\vec{a}\rangle \simeq-\varepsilon[20]$. 
TABLE I. Experimental parameters. $\Omega$ is the disk frequency of rotation, $\operatorname{Re}_{\lambda}=\left(15 v_{\text {rms }}^{4} / \nu \varepsilon\right)^{1 / 2}$, where $v_{\text {rms }}$ is the root-mean-square velocity defined as $v_{\text {rms }}=\sqrt{\left(v_{x}^{\prime 2}+v_{y}^{\prime 2}+v_{z}^{\prime 2}\right) / 3}$ (respectively, $\left.\sqrt{\left(v_{x}^{\prime 2}+2 v_{z}^{\prime 2}\right) / 3}\right)$ in the square (resp. round) geometry, $v_{s}=8.2 \times 10^{-6} \mathrm{~m}^{2} \mathrm{~s}^{-1}$ and $v_{r}=1.15 \times 10^{-6} \mathrm{~m}^{2} \mathrm{~s}^{-1}$ are the kinematic viscosities for the square and round geometries, and the dissipation rate $\varepsilon$ is estimated according to Refs. [20,33] for the square and round configurations, respectively. The Kolmogorov timescale is $\tau_{\eta}=(v / \varepsilon)^{1 / 2}$ and $t_{0 z}$ is the zero-crossing time of the acceleration autocorrelation function $\left.\left[R_{a_{z}}\left(t_{0, z}\right)=0\right)\right] . \tau_{a_{i}}$ are the acceleration component timescales measured from the integration of $R_{a_{i}}(\tau)$ up to the zero-crossing time (1) and Fig. 3(a), and their surrogates are defined: $\tau_{a i, s}^{\star}=C_{0 i}^{\star} \tau_{\eta} / 2 a_{0 i}$ (4) where $C_{0}{ }^{\star}$ and $C_{0}$ are distinguished by peak values in $d D_{2}^{L} / \mathrm{d} \tau \varepsilon^{-1}$ and $D_{2}^{L}(\varepsilon \tau)^{-1}$, respectively.

\begin{tabular}{lcccccccccccccc}
\hline \hline$\Omega$ & $\operatorname{Re}_{\lambda}$ & Geometry & $\begin{array}{c}v_{\text {rms }} \\
\left(\mathrm{m} \mathrm{s}^{-1}\right)\end{array}$ & $\begin{array}{c}\varepsilon \\
\left(\mathrm{m}^{2} \mathrm{~s}^{-3}\right)\end{array}$ & $\begin{array}{c}\tau_{\eta} \\
(\mathrm{ms})\end{array}$ & $\begin{array}{c}t_{0 z} / \tau_{\eta} \\
\mathrm{Hz}\end{array}$ & $\begin{array}{c}\left(\tau_{a x} / \tau_{a z}\right) / \\
\left(\tau_{a x, z}^{\star} / \tau_{a z, s}^{\star}\right)\end{array}$ & $C_{0 z}^{\star}$ & $C_{0 x}^{\star} / C_{0 z}^{\star}$ & $C_{0 z}$ & $C_{0 x} / C_{0 z}$ & $a_{0 z}$ & $a_{0 x} / a_{0 z}$ \\
\hline 4.2 & 140 & Square & 0.34 & 1.2 & 2.6 & 2.5 & 1.03 & 3.1 & 1.8 & 2.5 & 1.9 & 1.3 & 1.6 \\
5.5 & 180 & Square & 0.45 & 2.3 & 1.9 & 2.3 & 1.02 & 3.2 & 1.8 & 2.6 & 1.9 & 1.5 & 1.6 \\
6.9 & 200 & Square & 0.56 & 4.4 & 1.4 & 2.3 & 1.07 & 3.4 & 1.7 & 2.8 & 1.8 & 1.6 & 1.6 \\
4.1 & 450 & Round & 0.50 & 4.0 & 0.5 & 2.6 & 1.04 & 3.6 & 1.5 & 3.0 & 1.5 & 1.9 & 1.4 \\
6.4 & 630 & Round & 0.74 & 10.2 & 0.4 & 2.0 & 0.99 & 5.1 & 1.5 & 4.3 & 1.5 & 3.3 & 1.4 \\
7.2 & 730 & Round & 0.87 & 13.9 & 0.3 & 2.0 & 1.01 & 5.8 & 1.4 & 4.9 & 1.5 & 4.2 & 1.1 \\
8.5 & 810 & Round & 1.02 & 21.8 & 0.2 & 2.5 & 1.01 & 6.2 & 1.3 & 5.3 & 1.4 & 4.4 & 1.1 \\
\hline \hline
\end{tabular}

\section{ANISOTROPY IN THE ACCELERATION COMPONENT TIMESCALES}

The experimental techniques introduced in the previous section yield separate ensembles of trajectories: velocity tracks with unknown positions in the case of eLDV and differentiable position tracks in the case of SPTV. In all cases, acceleration signals are then obtained by finite difference schemes which lead to an amplification of experimental noise. Typically this noise is removed by convolution with Gaussian filters [45], which requires a subjective choice of the filter width. This choice poses a problem as a portion of the true signal may be removed when the filter width is chosen to be too wide, or too much noise may be retained when the filter width is chosen to be too narrow. To avoid such complications, we used a variable time step method [44] which permits an objective estimate of the acceleration components' covariance $\left(\left\langle a_{i}(t) a_{i}(t+\tau)\right\rangle\right)$ and variance $\left(\left\langle a_{i}^{2}\right\rangle\right)$ from which we compute $a_{0 i}$ using the Heisenberg-Yaglom relation: $\left\langle a_{i}^{2}\right\rangle=a_{0 i} \varepsilon^{3 / 2} v^{-1 / 2}$. The values of this nondimensional function are given in Table I and are similar to those obtained in previous works [22].

The Lagrangian velocity structure function $\left\langle[v(t+\tau)-v(t)]^{2}\right\rangle$, which we will refer to as $D_{2}^{L}(\tau)$ in what follows, is normalized by $v_{\mathrm{rms}}^{2}$ in Fig. 2 (a) and presents three regimes. First is a ballistic regime which extends up to times of the order of $\tau_{\eta}$ for which $D_{2}^{L}(\tau) \simeq\left\langle a^{2}\right\rangle \tau^{2}$. As such, the anisotropy in this regime is accurately predicted by the ratio of the acceleration variances given in Fig. 2(b). An asymptotic regime also exists when $\tau / \tau_{\eta}>20$ and the velocities at two times separated by $\tau$ become uncorrelated such that $D_{2}^{L}(\tau) \simeq\left\langle v^{2}\right\rangle$. The curves in Fig. 2(a) do not resolve this regime as trajectories are restricted to a small homogeneous region of the flow, but we expect the velocity variance anisotropy in Fig. 2(b) to be a satisfactory estimate of this asymptotic state. Finally, an intermediate regime exists for which $D_{2}^{L}(\tau) \simeq C_{0} \varepsilon \tau$. The structure function compensated by $\varepsilon \tau$ as well as a dimensionally similar, though kinematically distinct, normalization are plotted in Fig. 2(c); the latter is discussed comprehensively in the discussion pertaining to Eq. (3) below. Differing component-wise peak values in $D_{2}^{L}(\tau) /(\varepsilon \tau)=C_{0}$ are indicative of the large-scale anisotropy which strongly affects the Lagrangian velocity increment statistics in the inertial range. The values and trends measured here (Table I) for $C_{0}$ and $a_{0}$ are similar to those reported elsewhere [21] and are briefly summarized below.

Both values of $a_{0 i}$ and $C_{0 i}$ depend on the component and are increasing functions of the Reynolds number but with different dependencies. The ratio $C_{0 x} / C_{0 z}$ is always found to be larger than one, 



FIG. 2. Investigation of Lagrangian structure functions. (a) Second-order structure functions for the $x$ (०) and $z(\triangle)$ components are normalized by $v_{\mathrm{rms}}^{2}$ for $\operatorname{Re}_{\lambda}=140$. Dashed lines refer to the acceleration integral scale. (b) Ratios of anisotropy versus Reynolds number for velocity and acceleration variances. (c) Compensated structure functions where $(\bullet, \mathbf{\Delta})$ are the $(x, y)$ components of $D_{2}^{L}(\tau \varepsilon)^{-1}$ and $(\circ, \triangle)$ correspond to $d D_{2}^{L} / d \tau \varepsilon^{-1}$ at $\operatorname{Re}_{\lambda}$. Dashed lines correspond to the zero-crossing timescales.

while $a_{0 x} / a_{0 z}$ is closer to one in the high Reynolds number limit. This observation is related to the velocity fluctuations in the inertial scale range, which remain anisotropic [21], while small-scale quantities, such as the acceleration, are expected to be more isotropic as energy is redistributed between the different directions in the dissipative scale range. DNS results have demonstrated that the anisotropy in velocity and acceleration magnitude is due to the stagnation point topology of a strain-dominated flow so that acceleration (resp. velocity) is always slightly (resp. much) larger along the contracting direction $(x)$ when compared to the same quantity measured along the dilating direction $(z)$ [26]. We confirm that these trends are also present in the velocity and acceleration variances; Fig. 2(b) demonstrates that the acceleration anisotropy tends towards unity while the velocity remains strongly anisotropic, even at high Reynolds numbers as observed in Ref. [22]. Given the hierarchy of velocity and acceleration magnitudes and assuming the sole presence of dissipative contributions, it could be expected that the timescale of the acceleration component with the most active component would have the smallest correlation time. However, the opposite is true due to the presence of large-scale contributions, as we demonstrate below.

To investigate the temporal dynamics at small scale, we calculate the component-wise acceleration autocorrelation function, $R_{a_{i}}(\tau)=\left\langle a_{i}(t) a_{i}(t+\tau)\right\rangle /\left\langle a_{i}^{2}\right\rangle$, in Fig. 3(a). An anisotropy between the axial $(z)$ and radial $(x)$ components is apparent, with the latter decorrelating more slowly than the former. This anisotropy, characterized by a longer timescale for the most active component, was observed at all Reynolds numbers and is consistent with measurements in a similar apparatus with a round cross section at $\operatorname{Re}_{\lambda}=690$ [46] where the zero crossing of $R_{a_{i}}(\tau)$ yields $t_{0 x} / t_{0 z}=1.4$ and $t_{0 z} \simeq 2.2 \tau_{\eta}$. As can be seen in Fig. 3(a, inset) the zero-crossing anisotropy occurring in the present measurements is close to these values, $t_{0 x} / t_{0 z}=1.3 \pm 0.1$ and $t_{0 z} / \tau_{\eta}=2.3 \pm 0.2$ on average (Table I). An alternative estimate of the acceleration timescales, which will be of interest below, is obtained by integrating the positive part of the autocorrelation functions:

$$
\tau_{a_{i}}=\int_{0}^{t_{0 i}} R_{a_{i}}(\tau) d \tau .
$$

We note that the upper bound of Eq. (1) is not taken asymptotically as the integral of $R_{a i}(\tau)$ is the sum of the positive and negative lobes of Fig. 3(a) and is zero for homogogeneous and stationary turbulence [47]. The anisotropy, as measured using this integral timescale, is plotted in the inset of Fig. 3(a). Similar to the ratio of the zero crossings, we find $\tau_{a x} / \tau_{a z}=1.2 \pm 0.1$ with no evident Reynolds number dependence, which shows that all timescales evolve in the same manner with $\operatorname{Re}_{\lambda}$ irrespective of the acceleration component. To investigate the Reynolds number dependence 
(a)

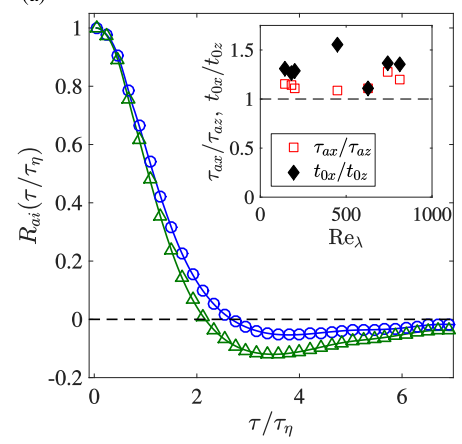

(b)



(c)

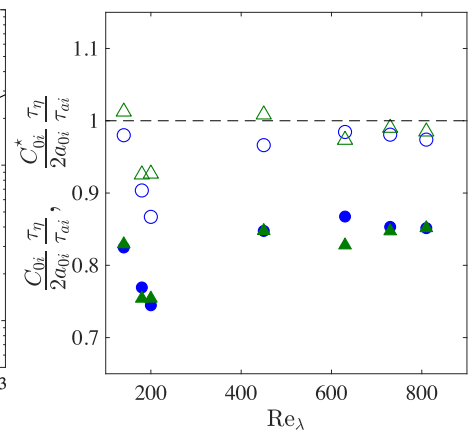

FIG. 3. (a) Acceleration autocorrelation functions $R_{a_{i}}(\tau)=\left\langle a_{i}(t) a_{i}(t+\tau)\right\rangle /\left\langle a_{i}^{2}\right\rangle$ of acceleration components $a_{x}(\circ)$ and $a_{z}(\triangle)$ for $\operatorname{Re}_{\lambda}=180$ where one in five symbols has been plotted for clarity. (Inset) Ratio of acceleration timescales: $\tau_{a_{x}} / \tau_{a_{z}}(\square), t_{0, x} / t_{0, z}(\diamond)$. (b) Ratios of the integral timescales $T_{E}=u^{\prime 2} / \varepsilon$ to the acceleration timescales as a function of the Reynolds number $\operatorname{Re}_{\lambda}: T_{E} / \tau_{a_{x}}(\circ), T_{E} / \tau_{a_{z}}(\triangle), T_{E} / t_{0, x}(\bullet)$, $T_{E} / t_{0, z}(\mathbf{\Delta})$, and $T_{E} / \tau_{\eta}(\times)$. Dashed line is the HIT prediction $T_{E} / \tau_{\eta}=\operatorname{Re}_{\lambda} / \sqrt{15}$. (c) Ratios of the measured acceleration timescales $\tau_{a i}$ to their surrogates $\tau_{a i}^{s}=C_{0 i}^{\star} \tau_{\eta} / 2 a_{0 i}$ as a function of the Taylor-scale Reynolds number, $i=x(\mathrm{O})$, and $i=z(\triangle)$; the Sawford jointly Gaussian two-time prediction of the acceleration integral timescale in the large Reynolds number limit (see the Appendix for details) is given by $i=x(\bullet)$ and $i=z(\boldsymbol{\Delta})$.

of $t_{0}$ and $\tau_{a}$, their behavior is compared with that predicted by $\mathrm{K} 41$, which gives the ratio of the energy containing to the dissipative scales: $T_{E} / \tau_{\eta}=\mathrm{Re}_{\lambda} / \sqrt{15}$ [48] where $T_{E}=v_{\mathrm{rms}}^{2} / \varepsilon$. As displayed in Fig. 3(b), we observe that the experimental measurement of $T_{E} / \tau_{\eta}$ is in agreement with the theoretical prediction even though the flow is neither isotropic nor homogeneous near the stagnation point of the central region. We observe that the ratio $T_{E} / \tau_{a_{i}}$ is not proportional to $\operatorname{Re}_{\lambda}$ although the proportionality holds to within a numerical factor for the case of zero-crossing times $t_{0 i}$, which may be expected as empirical evidence suggests that $t_{0 z} \simeq 2.2 \tau_{\eta}$ and that the anisotropy in $t_{0 x} / t_{0 z}$ is roughly constant with Reynolds number.

A jointly Gaussian two-time stochastic model introduced by Sawford [25] is known to reproduce the general shape of the acceleration autocorrelation curve. In this particular case for which the autocorrelation function may be calculated analytically it can be shown that the acceleration integral timescale can be expressed as the surrogate relation:

$$
\tau_{a, s}=\frac{C_{0}}{2 a_{0}} \tau_{\eta}
$$

which is expected to hold to within $5 \%$ error for the Reynolds numbers considered (see the discussion in the Appendix). This expression is tested experimentally in Fig. 3(c), and it is found for both axial and radial components that $\left(C_{0 i} \tau_{\eta} / 2 a_{0 i}\right) / \tau_{a i}=0.8$ holds to within a few percent. However, the coefficient 0.8 indicates $C_{0}$ may not be the proper nondimensional constant.

The mismatch of nearly $20 \%$ found in the prediction of the acceleration integral scale can be accounted for by studying the kinematic relationship relating the acceleration autocorrelation function to the velocity increments. Dropping the index $i$ for clarity, this reads

$$
\frac{d}{d \tau}\left\langle[v(t+\tau)-v(t)]^{2}\right\rangle=2\left\langle a^{2}\right\rangle \int_{0}^{\tau} R_{a}\left(\tau^{\prime}\right) d \tau^{\prime} .
$$

If a Lagrangian inertial range-type scaling is assumed to hold, we obtain $d D_{2}^{L} / d \tau=C_{0}^{\star} \varepsilon$ for the lefthand side of Eq. (3). This constraint is verified only when the integral on the right-hand side obtains a maximum, which occurs for $\tau=t_{0}$, the time which separates the positive part $\left(\tau \leqslant t_{0}\right)$ and the negative part ( $\left.\tau \geqslant t_{0}\right)$ of $R_{a}(\tau)$. We verify Eq. (3) in Fig. 2(c) where each vertical line corresponds 
to a component's zero crossing and passes through the corresponding peaks of $\varepsilon^{-1} d D_{2}^{L}(\tau) / d \tau$. The location of $t_{0}$ gives the time of the maximum rate of increase in the structure function, which corresponds to the end of the ballistic regime: $D_{2}^{L}(\tau) \simeq\left\langle a^{2}\right\rangle \tau^{2}$. The integral scale $\tau_{a}$, the second term on the right-hand-side of Eq. (3), is found to be at smaller timescales in the ballistic region than $t_{0}$ as seen in the vertical lines of Fig. 2(a). Interestingly, the solid symbols of Fig. 2(c) which correspond to $D_{2}^{L}(\tau) /(\varepsilon \tau)$ do not coincide with the zero crossings and their peaks are lower than those corresponding to $\varepsilon^{-1} d D_{2}^{L}(\tau) / d \tau$. We thus distinguish between the Kolmogorov coefficient calculated with the former $\left(C_{0}\right)$ and the latter $\left(C_{0}^{\star}\right)$ which is roughly $20 \%$ larger.

Combining the inertial range relation and Eq. (3), we define a second surrogate of the integral scale $\tau_{a}$, notated $\tau_{a, s}^{\star}$, from the relation $C_{0}^{\star} \varepsilon=2\left\langle a^{2}\right\rangle \tau_{a}^{s}$. Using the Heisenberg-Yaglom relation $\left\langle a^{2}\right\rangle=$ $a_{0} \varepsilon^{3 / 2} v^{-1 / 2}$, the surrogate $\tau_{a, s}^{\star}$ follows the scaling relation

$$
\tau_{a, s}^{\star}=\frac{C_{0}^{\star}}{2 a_{0}} \tau_{\eta}
$$

where $C_{0}^{\star}$ follows similar trends to $C_{0}$ and we recall that both $C_{0}$ and $a_{0}$ are increasing functions of $\operatorname{Re}_{\lambda}$ with different scalings. This surrogate expression for the acceleration integral scale is plotted in Fig. 3(c), and it accurately predicts $\tau_{a}$ to within a few percent without any numerical prefactor. In comparing the relationships in Fig. 3(c) it was found that $\left(C_{0 i} \tau_{\eta} / 2 a_{0 i}\right) / \tau_{a i}=0.8$ while $\left(C_{0 i}^{\star} \tau_{\eta} / 2 a_{0 i}\right) / \tau_{a i}=1$. The surrogate relationships calculated with $C_{0}^{\star}$ as opposed to $C_{0}$ gives different coefficients which correspond to the nearly $20 \%$ increase in peak values observed in Fig. 2(c) when calculating $d D_{2}^{L} / d \tau \varepsilon^{-1}$ as opposed to $D_{2}^{L}(\tau)(\varepsilon \tau)^{-1}$.

It appears that the two methods for calculating constants related to the inertial range are not the same, and the former is preferable as it is directly related to the velocity increments by the kinematic relationship (3) and leads to an accurate prediction of the acceleration integral scale. In the context of the Sawford model, asymptotically large Reynolds numbers lead the acceleration to behave as a delta-correlated white noise, and the second-order model reduces to a first-order Langevin equation $[25,41]$. The velocity autocorrelation in this case is an exponential function that is coherent with the inertial range scaling of the second-order structure function. In this regime we obtain $C_{0}^{\star}=C_{0}$ from Eq. (3) by replacing the acceleration covariance by the delta-correlated white noise of magnitude $C_{0} \varepsilon$.

Interestingly, for each Reynolds number experiment the transverse and axial directions give near equal values even though the shapes of the autocorrelation curves are different. Furthermore, the scaling $\tau_{a x} / \tau_{a z}=\left(C_{0 x}^{\star} / a_{0 x}\right) /\left(C_{0 z}^{\star} / a_{0 z}\right)$ reproduces the small timescale anisotropy to within at most $7 \%$ (Table I). These observations suggest that the changing shape of the autocorrelation curves, evident in Fig. 3(a), depends on the constants $C_{0}^{\star}$ and $a_{0}$. Finally, we note that the relation $\tau_{a}=$ $C_{0}^{\star} \tau_{\eta} /\left(2 a_{0}\right)$ leads to an interesting prediction concerning the anisotropy of acceleration timescales in the limit of asymptotically high Reynolds numbers. As both $C_{0}^{\star}$ and $a_{0}$ converge toward finite values with $\left(C_{0, x}^{\star} / a_{0, x}\right) /\left(C_{0, z}^{\star} / a_{0, z}\right) \simeq 1.2-1.4$ in this limit (see Ref. [21] and Table I), the acceleration timescale is expected to remain anisotropic, even at high Reynolds numbers.

The apparent contradiction between the observed anisotropic small-scale fluctuations and the K41 hypothesis of local isotropy in fully turbulent flow [23] is addressed by investigating the scaleby-scale distribution of energy for Lagrangian acceleration. Indeed, it was shown above that the acceleration timescale has two main contributions: first, from inertial range motions determined by $C_{0}$ and, second, from dissipative range motions characterized by $a_{0}$. To compare these contributions we investigated the power spectrum of acceleration components, $\phi_{a i}$, computed from the collection of twice differentiated position tracks from the SPTV data set using Welch's method [49]. The spectrum depends on angular frequency $\omega$ and is formally related to the acceleration autocorrelation function by the Wiener-Khinchin theorem [50,51]:

$$
\phi_{a_{i}}(\omega)=2 \int_{0}^{\infty} R_{a_{i}}(\tau) e^{-i \omega \tau} d \tau .
$$




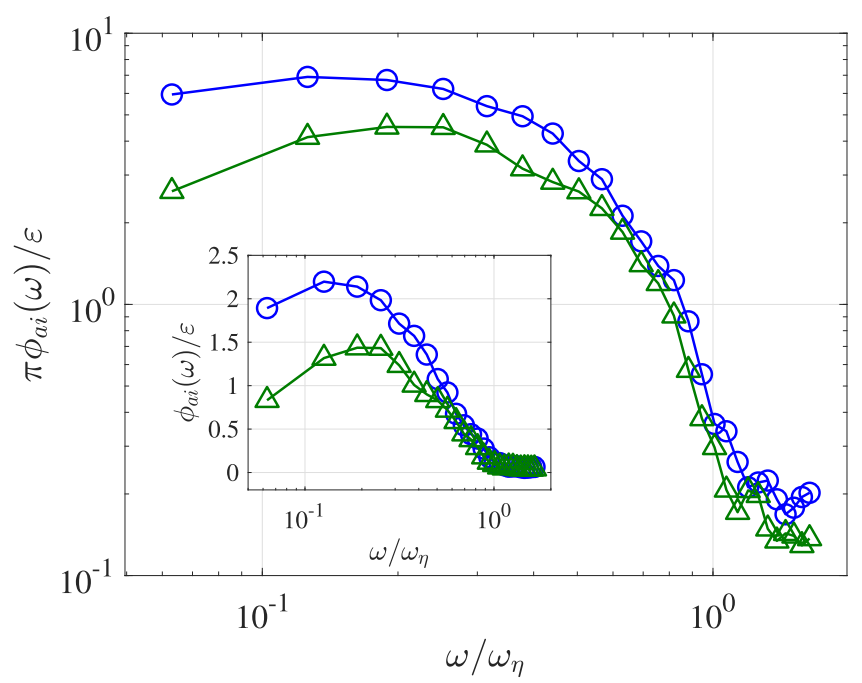

FIG. 4. Normalized power spectra of acceleration components, $\pi \phi_{a x}\left(\omega / \omega_{\eta}\right) / \varepsilon(\circ)$ and $\pi \phi_{a z}\left(\omega / \omega_{\eta}\right) / \varepsilon(\triangle)$, where the angular frequency is normalized by $\omega_{\eta}=\pi / \tau_{\eta}$ for $\operatorname{Re}_{\lambda}=180$. Each spectrum is the ensemble average of raw spectra computed with unfiltered position tracks which are differentiated twice, leading to noise amplification in the high-frequency range. (Inset) Modified scaling in semilog coordinates, with same symbols.

The acceleration spectra are plotted in Fig. 4 as a function of $\omega / \omega_{\eta}$ where $\omega_{\eta}=\pi / \tau_{\eta}$ is the Kolmogorov angular frequency. They have been normalized by $\varepsilon \pi^{-1}$, which has been used in the literature to account for Reynolds number dependence [21,23,52]. Each spectrum is mainly composed of three regions:

(1) A horizontal plateau in the low-frequency region $\omega \leqslant 0.3 \omega_{\eta}$, corresponding to uncorrelated fluctuations. The plateau values obtained here are in good agreement with those found in the literature, and in Fig. 4 (inset) the spectra are given in log-linear coordinates using the modified scaling $\varepsilon^{-1}$ preferred by some authors [21,24,53].

(2) A cutoff region situated in the near-dissipative range $\omega \approx \omega_{\eta}$ where the spectrum decreases rapidly.

(3) A high-frequency region $\omega \geqslant \omega_{\eta}$ where the spectrum flattens out due to double derivation of the small white noise contained in the position tracks. This region is visible because the spectra presented here are raw spectra obtained with unfiltered data. Note that spectra extend only down to $0.06 \omega_{\eta}$ as we restrict the trajectories to a small homogeneous region with a volume of $1 \mathrm{~cm}^{3}$ near the stagnation point to avoid any effect due to the spatial nonhomogeneity of the flow.

Figure 4 shows that anisotropy is contained in the low frequencies below $\omega \leqslant 0.3 \omega_{\eta}$ and decreases as frequency increases such that the acceleration spectra are almost identical in the dissipative region $\left(\omega \geqslant 0.6 \omega_{\eta}\right)$. It is seen from this figure that there is no real contradiction between isotropy at small scale and the anisotropy of small-scale statistical quantities such as acceleration variance or acceleration timescale $\tau_{a}$. Both integral quantities have a contribution from low frequencies, i.e., from scales larger than the dissipative scales, which are anisotropic, and these contributions do not become negligible in the limit of high Reynolds numbers.

\section{CONCLUSION}

We have investigated the anisotropy of turbulent acceleration signals in a flow subject to large-scale straining and presented scalings that accurately predict the results based on K41 phenomenology and a jointly Gaussian two-time stochastic model. Our observation that the strongest 
acceleration component decorrelates more slowly than the weakest component is in agreement with other experiments and simulations in the literature. These results complement early work measuring the anisotropy in the Lagrangian constants $C_{0}$ [21] and $a_{0}$ [22,29] in that both constants contribute to the acceleration timescale $\tau_{a}=\int_{0}^{t_{0}} R_{a}(\tau) d \tau$, which is well estimated by $\tau_{a, s}=0.8 C_{0} \tau_{\eta} / 2 a_{0}$, a result derived from the jointly Gaussian two-time stochastic model introduced by Sawford [25]. A second, complementary timescale results from a kinematic relationship directly relating the velocity increments to the acceleration integral scale as $\tau_{a, s}^{\star}=C_{0}^{\star} \tau_{\eta} / 2 a_{0}$ where the constant $C_{0}^{\star}$ is preferable to $C_{0}$ as it is explicitly related to the velocity increments. This relationship predicts the anisotropy of the integral timescales to within at most $7 \%$ over nearly a decade of variation in the Taylor-scale Reynolds numbers $\operatorname{Re}_{\lambda}$. As both Lagrangian second-order structure function scaling constants $C_{0}$ and $C_{0}{ }^{\star}$ are expected to remain anisotropic while $a_{0}$ tends toward isotropy, both $\tau_{a, s}$ and $\tau_{a, s}^{\star}$ predict that acceleration timescales will remain anisotropic in the high-Reynolds limit. However, this prediction does not contradict common intuition that small-scale motions should become isotropic as is confirmed by investigating dissipative-scale fluctuations in the acceleration power spectra, which are indeed isotropic. We note that the expressions for the integral scale were not derived solely on the grounds of a proposed turbulence phenomenology, but are more likely due to the nature of Lagrangian trajectories, which have two characteristic, well-separated, timescales. The surrogate acceleration integral scale $\tau_{a, s}^{\star}$ is expected to hold for other turbulent flows in the fully developed regime and should be tested both experimentally and numerically.

\section{ACKNOWLEDGMENTS}

This work is supported by Agence Nationale de la Recherche (Grant No. ANR-13BS09-0009). Financial support from the project IDEXLYON at the University of Lyon within the framework of the French program "Programme Investissements d'Avenir" (ANR-16-IDEX-0005) is also acknowledged.

\section{APPENDIX: ACCELERATION TIMESCALE IN THE CASE OF A JOINTLY GAUSSIAN TWO-TIME MODEL}

Below we derive the relation $\tau_{a} \simeq C_{0} \tau_{\eta} / 2 a_{0}$ from Sawford's jointly Gaussian two-time model of the Lagrangian turbulent velocity [25]. This model has been shown to qualitatively reproduce the shape of velocity and acceleration correlation functions [25,32,52], and we recall below the main results of the model before establishing the result. A reader familiar with the notations introduced of Sawford's article may start directly with expression (A4) for the acceleration correlation function $R_{a}(\tau)$.

In Sawford's jointly Gaussian two-time model, the Lagrangian velocity $u$ and its forcing $f$ are defined as jointly Gaussian stochastic processes. Using the formulation given in the Appendix of Ref. [25], equivalent to that of Krasnoff [54], $u$ and $f$ are solutions of the stochastic differential equations:

$$
\begin{gathered}
d u=\beta_{1} u d t+f d t, \\
d f=\beta_{2} f d t+\sqrt{-2 \beta_{2}\left\langle f^{2}\right\rangle} d W
\end{gathered}
$$

where $-1 / \beta_{1}$ is a long timescale (of the order of the Lagrangian integral time $T_{L}$ ), $-1 / \beta_{2}$ a short timescale (further proved to be close to $\tau_{a}$ ), and $d W$ a delta-correlated Gaussian noise satisfying the relation $\left\langle d W(t) d W\left(t^{\prime}\right)\right\rangle=\delta\left(t-t^{\prime}\right)$. In this model, velocity is differentiable so that acceleration $a=d u / d t$ exists, and the velocity variance is linked to the forcing variance by the relation 
$\left\langle f^{2}\right\rangle=\left(\beta_{1}+\beta_{2}\right) \beta_{1}\left\langle u^{2}\right\rangle$. All flow properties are set by the triplet $\left(\left\langle u^{2}\right\rangle,-1 / \beta_{1},-1 / \beta_{2}\right)$, and velocity and acceleration correlation functions read

$$
\begin{aligned}
& R_{u}(\tau)=\frac{1}{\beta_{2}-\beta_{1}}\left[\beta_{2} \exp \left(\beta_{1}|\tau|\right)-\beta_{1} \exp \left(\beta_{2} \mid \tau\right) \mid\right], \\
& R_{a}(\tau)=\frac{1}{\beta_{2}-\beta_{1}}\left[\beta_{2} \exp \left(\beta_{2}|\tau|\right)-\beta_{1} \exp \left(\beta_{1}|\tau|\right)\right] .
\end{aligned}
$$

Matching the inertial and dissipation subrange as given by Kolmogorov's similarity theory gives $-1 / \beta_{1}=2\left\langle u^{2}\right\rangle /\left(C_{0} \varepsilon\right)=T_{L}$ and $-1 / \beta_{2}=C_{0} \tau_{\eta} / 2 a_{0}$.

Defining $t_{0}$ so that $R_{a}\left(t_{0}\right)=0$ and integrating $R_{a}(\tau)$ up to $t_{0}$, we obtain the acceleration integral timescale $\tau_{a}$ :

$$
\tau_{a}=\int_{0}^{t_{0}} R_{a}(\tau) d \tau=-\frac{\exp \left(\beta_{1} t_{0}\right)}{\beta_{2}} .
$$

In a high Reynolds number flow, it is expected that $\left|\beta_{1} t_{0}\right|=t_{0} / T_{L} \ll 1$ so that $\tau_{a} \simeq-1 / \beta_{2}=$ $C_{0} \tau_{\eta} / 2 a_{0}$. More precisely, for a flow with $\operatorname{Re}_{\lambda}=500$ (corresponding to $T_{L} / \tau_{\eta} \simeq 60$ [41]), one can then estimate $\exp \left(\beta_{1} t_{0}\right) \simeq 0.96$ so that $\tau_{a} \simeq C_{0} \tau_{\eta} / 2 a_{0}$ within $5 \%$ error. In the context of this model, the correlation time of the acceleration is equal to the characteristic timescale of the stochastic forcing.

[1] P. Sagaut and C. Cambon, Homogeneous Turbulence Dynamics (Cambridge University Press, Cambridge, 2008).

[2] L. Prandtl, Attaining a steady air stream in wind tunnels, NASA Technical Memo 726 (1933).

[3] G. K. Batchelor and I. Proudman, The effect of rapid distortion of a fluid in turbulent motion, Q. J. Mech. Appl. Math. 7, 83 (1954).

[4] G. I. Taylor, Turbulence in a contracting stream, Z. Angew. Math. Mech. 15, 91 (1935).

[5] S. A. Ayyalasomayajula and Z. Warhaft, Nonlinear interactions in strained axisymmetric high-Reynoldsnumber turbulence, J. Fluid Mech. 566, 273 (2006).

[6] M. P. Clay and P. K. Yeung, A numerical study of turbulence under temporally evolving axisymmetric contraction and subsequent relaxation, J. Fluid Mech. 805, 460 (2016).

[7] R. R. Mills and S. Corrsin, Effect of contraction in turbulence and temperature fluctuations generated by a warm grid, NASA Memo 5-5-59W (1959).

[8] A. J. Reynolds and H. J. Tucker, The distortion of turbulence by general uniform irrotational strain, J. Fluid Mech. 68, 673 (1975).

[9] A. A. Townsend, The uniform distortion of homogeneous turbulence, Q. J. Mech. Appl. Math. 7, 104 (1954).

[10] M. S. Uberoi, Effect of wind-tunnel contraction on free-stream, J. Aeronaut. Sci. 23, 754 (1956).

[11] M. S. Uberoi and S. Corrsin, Diffusion of heat from a line source in isotropic turbulence, N.A.C.A. Report, $1142(1953)$.

[12] S. Ott and J. Mann, An experimental investigation of the relative diffusion of particle pairs in threedimensional turbulent flow, J. Fluid Mech. 422, 207 (2000).

[13] N. Ouellette, H. Xu, and E. Bodenschatz, A quantitative study of three-dimensional Lagrangian particle tracking algorithms, Exp. Fluids 40, 301 (2006).

[14] Y. Sato and K. Yamamoto, Lagrangian measurement of fluid-particle motion in an isotropic turbulent field, J. Fluid Mech. 175, 183 (1987).

[15] B. L. Sawford and P. K. Yeung, Eulerian acceleration statistics as a discriminator between Lagrangian stochastic models in uniform shear flow, Phys. Fluids 12, 2033 (2000).

[16] P. Shen and P. K. Yeung, Fluid particle dispersion in homogeneous turbulent shear flow, Phys. Fluids 9 , 3472 (1997). 
[17] P. Yeung and S. Pope, An algorithm for tracking fluid particles in numerical simulations of homogeneous turbulence, J. Comput. Phys. 79, 373 (1988).

[18] L. Marié and F. Daviaud, Experimental measurement of the scale-by-scale momentum transport budget in a turbulent shear flow, Phys. Fluids 16, 457 (2004).

[19] F. Ravelet, A. Chiffaudel, and F. Daviaud, Supercritical transition to turbulence in an inertially driven von Kármán closed flow, J. Fluid Mech. 601, 339 (2008).

[20] P. D. Huck, N. Machicoane, and R. Volk, Production and dissipation of turbulent fluctuations close to a stagnation point, Phys. Rev. Fluids 2, 084601 (2017).

[21] N. T. Ouellette, H. Xu, M. Bourgoin, and E. Bodenschatz, Small-scale anisotropy in Lagrangian turbulence, New J. Phys. 8, 102 (2006).

[22] G. A. Voth, A. La Porta, A. M. Crawford, J. Alexander, and E. Bodenschatz, Measurement of particle accelerations in fully developed turbulence, J. Fluid Mech. 469, 121 (2002).

[23] A. S. Monin and A. M. Yaglom, Statistical Fluid Mechanics, Vol. II (MIT Press, Cambridge, MA, 1975).

[24] R. C. Lien and E. A. D'Asaro, The Kolmogorov constant for the Lagrangian velocity spectrum and structure function, Phys. Fluids 14, 4456 (2002).

[25] B. L. Sawford, Reynolds number effects in Lagrangian stochastic models of turbulent dispersion, Phys. Fluids 3, 1577 (1991).

[26] C.-M. Lee, A. Gylfason, P. Perlekar, and F. Toschi, Inertial particle acceleration in strained turbulence, J. Fluid Mech. 785, 31 (2015).

[27] W. Heisenberg, Zur statistischen Theorie der Kernreaktionen, Z. Phys. 124, 628 (1948).

[28] A. Yaglom, On acceleration fields in a turbulent flow, Dokl. Akad. Nauk. SSSR 69, 531 (1949).

[29] A. La Porta, G. A. Voth, A. M. Crawford, J. Alexander, and E. Bodenschatz, Fluid particle accelerations in fully developed turbulence, Nature (London) 409, 1017 (2001).

[30] A. M. Crawford, N. Mordant, and E. Bodenschatz, Joint Statistics of the Lagrangian Acceleration and Velocity in Fully Developed Turbulence, Phys. Rev. Lett. 94, 024501 (2005).

[31] P. K. Yeung and S. B. Pope, Lagrangian statistics from direct numerical simulations of isotropic turbulence, J. Fluid Mech. 207, 531 (1989).

[32] N. Mordant, E. Leveque, and J.-F. Pinton, Experimental and numerical study of the Lagrangian dynamics of high Reynolds turbulence, New J. Phys. 6, 116 (2004).

[33] R. Volk, E. Calzavarini, E. Leveque, and J.-F. Pinton, Dynamics of inertial range particles in a turbulent flow, J. Fluid Mech. 668, 223 (2011).

[34] R. Volk, N. Mordant, G. Verhille, and J.-F. Pinton, Laser Doppler measurement of inertial particle and bubble accelerations in turbulence, Europhys. Lett. 81, 34002 (2008).

[35] P. Yeung, One- and two-particle Lagrangian acceleration correlations in numerically simulated homogeneous turbulence, Phys. Fluids 9, 2981 (1997).

[36] P. K. Yeung, S. B. Pope, E. A. Kurth, and A. G. Lamorgese, Lagrangian conditional statistics, acceleration and local relative motion in numerically simulated isotropic turbulence, J. Fluid Mech. 582, 399 (2007).

[37] N. Stelzenmuller, J.-I. Polanco, L. Vignal, I. Vinkovic, and N. Mordant, Lagrangian acceleration statistics in a turbulent channel flow, Phys. Rev. Fluids 2, 054602 (2017).

[38] L. Del Castello and H. J. H. Clercx, Lagrangian Acceleration of Passive Tracers in Statistically Steady Rotating Turbulence, Phys. Rev. Lett. 107, 214502 (2011).

[39] P. Huck, N. Machicoane, and R. Volk, A cost-efficient shadow particle tracking velocimetry setup suitable for tracking small objects in a large volume, Procedia IUTAM 20, 175 (2017).

[40] N. Machicoane, J. Bonaventure, and R. Volk, Melting dynamics of large ice balls in a turbulent swirling flow, Phys. Fluids 25, 125101 (2013).

[41] J.-F. Pinton and B. L. Sawford, A Lagrangian View of Turbulent Dispersion and Mixing (Cambridge University Press, Cambridge, 2012).

[42] R. D. Brown, Z. Warhaft, and G. A. Voth, Acceleration Statistics of Neutrally Buoyant Spherical Particles in Intense Turbulence, Phys. Rev. Lett. 103, 194501 (2009).

[43] G. Zocchi, P. Tabeling, J. Maurer, and H. Willaime, Measurement of the scaling of dissipation at high Reynolds numbers, Phys. Rev. E 50, 3693 (1994). 
[44] N. Machicoane, P. D. Huck, and R. Volk, Estimating two-point statistics from derivatives of a signal containing noise: Application to auto-correlation functions of turbulent Lagrangian tracks, Rev. Sci. Instrum. 88, 065113 (2017).

[45] N. Mordant, A. M. Crawford, and E. Bodenschatz, Experimental Lagrangian acceleration probability density function measurement, Physica D 193, 245 (2004).

[46] N. Mordant, A. M. Crawford, and E. Bodenschatz, Three-Dimensional Structure of the Lagrangian Acceleration in Turbulent Flows, Phys. Rev. Lett. 93, 214501 (2004).

[47] M. S. Borgas and B. L. Sawford, The small-scale structure of acceleration correlations and its role in the statistical theory of turbulent dispersion, J. Fluid Mech. 228, 295 (1991).

[48] H. Tennekes and J. Lumley, A First Course in Turbulence (MIT Press, Cambridge, MA, 2000).

[49] P. Welch, The use of fast Fourier transform for the estimation of power spectra: A method based on time averaging over short, modified periodograms, IEEE Trans. Audio Electroacoust. 15, 70 (1967).

[50] A. Khintchine, Korrelationstheorie der stationaren stochastischen Prozesse, Math. Ann. 109, 604 (1934).

[51] N. Wiener, Generalized harmonic analysis, Acta Math. 55, 117 (1930).

[52] B. L. Sawford and P. K. Yeung, Kolmogorov similarity scaling for one-particle lagrangian statistics, Phys. Fluids 23, 091704 (2011).

[53] R.-C. Lien, E. A. D'Asaro, and G. T. Dairiki, Lagrangian frequency spectra of vertical velocity and vorticity in high-Reynolds-number oceanic turbulence, J. Fluid Mech. 362, 177 (1998).

[54] E. Krasnoff and R. L. Peskin, The Langevin model for turbulent diffusion, Geophys. Fluid Dyn. 2, 123 (1971). 